Dermatology

DOI: $10.1159 / 000487080$

\section{Why We Should Focus on Melanoma-Targeted Screening Strategies}

Cédric Rat ${ }^{\mathrm{a}, \mathrm{b}}$ Brigitte Dreno ${ }^{\mathrm{b}, \mathrm{c}}$ Jean-Michel Nguyen ${ }^{\mathrm{b}, \mathrm{d}}$

a Department of General Practice, Faculty of Medicine, Nantes, France; ${ }^{b}$ French National Institute of Health and Medical Research/INSERM U1232, CRCINA team 2, Nantes, France; 'Oncodermatology Department, CHU Nantes, Nantes, France; dDepartment of Epidemiology and Biostatistics, CHU Nantes, Nantes, France

\section{Keywords}

Melanoma - Cancer screening - Early detection of cancer · Health inequality

There is no randomized controlled trial which formally proves that melanoma screening does more benefit than harm [1]. Due to the lack of evidence this is discussed very controversially and very emotionally. Jørgensen has recently commented a publication by Braun et al. [2] and recalled 3 major issues related to melanoma screening [3]. In order to further the debate, it is now mandatory to distinguish melanoma screening in the general population (individuals at average risk of melanoma) and targeted screening strategies focusing on high-risk populations.

(1) Determining whether melanoma screening is cost-effective is a major question. Melanoma screening in the general population at average risk of melanoma is expensive. The main reason is the low incidence rate of the disease. According to an individual perspective, determining whether a lesion is a melanoma (or not) is not expensive: diagnosis requires a clinical skin examination, dermoscopy and an excision - as reported by Braun et al. [2] - whereas early detection of other cancers requires more complex strategies.

In order to be cost-effective, further research on melanoma screening should focus on individuals at increased risk of melanoma. Our team has developed a targeted melanoma screening procedure grounded in primary care, involving individuals at elevated risk of melanoma selected using the Self-Assessment of Melanoma risk score (SAM score) [4-11]. The procedure, based both on a validated melanoma risk assessment [4-6] and on a GP skin examination, is promising: the incidence of melanoma was multiplied by more than 10 in the selected population [7-11]. This targeted screening strategy promises to be more efficient and less burdensome than screening the general population.

(2) Both Jørgensen and Braun discuss the potential harm of melanoma screening in the general population [2-3].

We agree that the number of false-positive results related to a melanoma screening in the population at average risk of melano- ma could be considerable. The main reason is the low incidence rate of the disease: basic statistics explain that the specificity of a screening procedure will decrease (and the number of false-positive results considerably increase), depending on whether the disease incidence is high (in selected populations) or low (in general unselected populations).

In order to improve the balance between benefits and harm, further research on melanoma screening should focus on individuals at increased risk of melanoma.

According to an individual perspective, we assume that the excision of a nevus which would appear benign after a pathological analysis is not a major harm: various patients obtain excision of nevi for functional or esthetic reasons and excisions of nevi are common.

(3) Do we screen the right people? Do we really reach out to the risk population?

Both Jørgensen and Braun report a major limitation of the EUROMELANOMA campaigns conducted in the general population at average risk of melanoma: only $24 \%$ of the participants $(263 / 1,087)$ agreed to be followed up [8-10]. Braun analyzed the major question as "whether we screen the right people, i.e. whether we really reach out to the risk population which we would otherwise miss." We assume that a major issue is health inequalities [11]. While significant funds are spent by policymakers to enhance melanoma awareness in the general population, patients are selfdirected to seek consultations. As a result, various populations (men, older patients, and socioeconomically disadvantaged populations) do not perform skin examination and have thick melanomas at the moment of diagnosis $[12,13]$. In total, melanoma exhibits the highest inequalities of all cancers when focusing on diagnosis at advanced stages [12]. In order to address this major issue, policymakers should promote the development of organized targeted melanoma screening programs, focusing on individuals at increased risk of melanoma.

In conclusion, screening campaigns in the general population - as reported by Braun et al. [2] - are not cost-effective, the balance between benefit and harm is unclear, and it does not address health inequality issues. A limit of developing no screening strategy [3] is that individuals consult on their own initiative, with no effect on health inequalities. We agree with Braun et al. that the development and validation of a new, tailored and more effective strategy is necessary to reduce inequalities at the screening stage. We invite researchers who would like to assess a melanoma-targeted screening intervention via an international randomized controlled trial to join a melanoma-targeted screening task force.

\section{Key Message}

Further research on melanoma screening should focus on individuals at increased risk of melanoma.

Disclosure Statement

The authors have no conflicts of interest to disclose.

\section{KARGER}

(C) 2018 S. Karger AG, Basel

E-Mail karger@karger.com

www.karger.com/drm
Cédric Rat

Department of General Practice, Faculty of Medicine of Nantes

1 , rue Gaston-Veil

FR-44035 Nantes (France)

E-Mail cedric.rat@univ-nantes.fr 


\section{References}

1 Johansson M, Brodersen J, Gøtzsche PC, et al: Screening for reducing morbidity and mortality in malignant melanoma. 2016. http://onlinelibrary.wiley.com/doi/10.1002/14651858.CD012352/full.

$\checkmark 2$ Braun RP, et al: Evaluation of the National Swiss Skin Cancer Screening Campaign 2013 - do we do the right thing? Dermatology 2017;233:404409.

3 Jørgensen KJ: Melanoma screening. Dermatology 2017;233:410-411.

4 Quéreux G, Nguyen JM, Volteau C, et al: Creation and test of a questionnaire for self-assessment of melanoma risk factors. Eur J Cancer Prev 2010;19:48-54

5 Quéreux G, Moyse D, Lequeux Y, et al: Development of an individual score for melanoma risk. Eur J Cancer Prev 2011;20:217-224.

-6 Quéreux G, Nguyen JM, Cary M, et al: Validation of the Self-Assessment of Melanoma Risk Score for a melanoma-targeted screening. Eur J Cancer Prev 2012;21:588-595.

-7 Rat C, Quéreux G, Rivière C, et al: Targeted melanoma prevention intervention: a cluster randomized controlled trial. Ann Fam Med 2014;12: $21-28$.
8 Rat C, Quéreux G, Grimault C, et al: Melanoma incidence and patient compliance in a targeted melanoma screening intervention. One-year follow-up in a large French cohort of high-risk patients. Eur J Gen Pract 2015;21:124-130.

-9 Rat C, Grimault C, Quéreux G, et al: Proposal for an annual skin examination by a general practitioner for patients at high risk for melanoma: a French cohort study. BMJ Open 2015;5:e007471.

10 Rat C, Quöreux G, Monégier du Sorbier M, et al: Patients at elevated risk of melanoma: individual predictors of non-compliance to GP referral for a dermatologist consultation. Prev Med 2014;64:48-53.

11 Rat C, Quéreux G, Grimault C, et al: Inclusion of populations at risk of advanced melanoma in an opportunistic targeted screening project involving general practitioners. Scand J Prim Health Care 2016;34:286294.

12 Lyratzopoulos G, Abel GA, Brown CH, et al: Socio-demographic inequalities in stage of cancer diagnosis: evidence from patients with female breast, lung, colon, rectal, prostate, renal, bladder, melanoma, ovarian and endometrial cancer. Ann Oncol 2013;24:843-850.

13 Birch-Johansen F, Hvilsom G, Kjaer T, et al: Social inequality and incidence of and survival from malignant melanoma in a population-based study in Denmark, 1994-2003. Eur J Cancer 2008;44:2043-2049. 